\title{
Neuropsychiatric manifestations in a child with moyamoya disease
}

Sir, Moyamoya disease (MMD) is a rare (prevalence of 3 cases/100,000 children), occlusive disorder of the cerebral vasculature. There is a progressive stenosis of terminal parts of bilateral internal carotid arteries and the main trunks of anterior and middle cerebral arteries, resulting in the formation of collateral vessels at the base of the brain. These small collateral vessels create "puff of smoke" appearance on angiogram. There are various clinical manifestations for MMD. There is bimodal age presentation with first peak occurring in the first decade of life, associated with cerebral infarction as progressive carotid occlusion develops. The adult patient most often present in the fourth decade with intracranial hemorrhage arising from rupture of the delicate network of collateral vessels which is mostly intraparenchymal and may be intraventricular or occasionally subarachnoid bleed.$^{[1]}$ In children, the most common presentation is that of recurrent episodes of cerebral ischemia manifesting clinically as focal deficits, paresthesia, and seizures. ${ }^{[2,3]}$

Psychiatric manifestations associated with MMD in children are uncommon. ${ }^{[4]}$ These include schizophrenia, ${ }^{[4]}$ acute transient psychosis, ${ }^{[5]}$ and mania. ${ }^{[6]}$ Some patients of MMD also show cognitive impairment, learning disability, and attention deficits.

We present a 14-year-old male child with features of focal seizures, hemicranial headache and certain behavioral problems in whom MMD was diagnosed through magnetic resonance imaging (MRI) and magnetic resonance angiography (MRA).

Master A, a 14-year-old male child with uneventful birth and developmental history without past and family history of neuro-psychiatric illness presented with complaints sudden abnormal movement of the right side of the body from last 5 years. These abnormal movements are a tonic in nature, recurrent, each lasting for about $5 \mathrm{~min}$, occurring at an interval of 1-2 months. There is no history of loss of consciousness. There is also a complaint of frequent left hemicranial headache from last 2 years. Along with this he has features of hyperactivity, easy distractibility, inattention, over-talkativeness, poor academic performance, forgetfulness in daily activities, irritability, stubbornness, emotional lability, temper tantrums from the last 4 years. 
On examination, the child was conscious, vitals were normal and there was no objective neurologic finding. The child was diagnosed to have focal seizures and was investigated. Investigations including a complete hemogram, liver and renal function tests, fasting blood glucose, lipid profile, serum ceruloplasmin, homocysteine, urine copper and abnormal metabolites, echocardiography were normal.

MRI and MRA showed bilateral loss of normal flow signal with cavernous and supra-clenoid segments indicating internal carotid artery occlusion, multiple collateral vascular channels along with the basal and suprasellar cistern region and along the basal ganglia, thalamus and capsular region, left centrum semi-ovale chronic lacunar infarcts. So bilateral supraclenoid internal carotid artery narrowing, prominent leptomeningial collateral branches and posterior circulation giving a puff of smoke appearance suggestive of MMD. Electroencephalogram (EEG) showed paroxysmal spikes and waves discharges from left fronto-temporal leads with generalization. Though he has features of hyperactivity and inattention, but on evaluation he does not fulfill the Diagnostic and Statistical Manual of Mental Disorders-V criteria for attention deficit hyperactivity disorder (ADHD). Psychological testing revealed IQ of 60 . The patient was started on tablet oxcarbazepine $600 \mathrm{mg} /$ day in divided doses. Neurosurgical opinion was sought. Surgical intervention, i.e., revascularization procedures (encephalo-duro-arterio-myo-synangiosis) was planned. The family members were psychoeducated about the behavioral problems of the patient.

Although the MMD is the most common in Japan, many subsequent cases have been reported elsewhere, including North America, Europe, and India. ${ }^{[7]}$ Information about the clinical features and long-term outcome of MMD in India is lacking. Indian patients with moyamoya showed a more benign natural history and a later age at presentation compared to East Asians. ${ }^{[8]}$

Although epilepsy is mentioned as an important manifestation of MMD, there are very few published reports. ${ }^{[3]}$ Our child with focal seizures and on the investigation, he was found to have abnormal EEG and findings suggestive of MMD on MRI brain with MRA.

Psychiatric manifestations associated with MMD have been reported in few published reports. In our patient, subsyndromal ADHD with certain other behavioral manifestations were observed. Tablet oxcarbazepine $600 \mathrm{mg} /$ day was useful in our patient for both control of seizures and behavioral problems.
MMD is an easily overlooked condition because of its low incidence and may not be considered as a possible diagnosis for the clinical presentation. Because of its progressive nature, it is imperative to diagnose MMD early and offer surgical treatment.

\section{Financial support and sponsorship \\ Nil.}

\section{Conflicts of interest}

There are no conflicts of interest.

Satyakam Mohapatra, Alok Jyoti Sahoo

Department of Psychiatry, S.C.B. Medical College, Mental Health Institute, Cuttack, Odisha, India

Address for correspondence: Dr. Satyakam Mohapatra,

Department of Psychiatry, S.C.B. Medical College, Mental Health Institute, Cuttack - 753 007, Odisha, India. E-mail: satyakgmu@gmail.com

\section{References}

1. Kitahara T, Ariga N, Yamaura A, Makino H, Maki Y. Familial occurrence of moya-moya disease: Report of three Japanese families. J Neurol Neurosurg Psychiatry 1979;42:208-14.

2. Vernet O, Montes JL, O'Gorman AM, Baruchel S, Farmer JP. Encephaloduroarterio - Synangiosis in a child with sickle cell anemia and moyamoya disease. Pediatr Neurol 1996;14:226-30.

3. Kuroda S, Houkin K. Moyamoya disease: Current concepts and future perspectives. Lancet Neurol 2008; 7:1056-66.

4. Lubman DI, Pantelis C, Desmond P, Proffitt TM, Velakoulis D. Moyamoya disease in a patient with schizophrenia. J Int Neuropsychol Soc 2003;9:806-10.

5. Klasen H, Britton J, Newman M. Moyamoya disease in a 12-year-old Caucasian boy presenting with acute transient psychosis. Eur Child Adolesc Psychiatry 1999;8:149-53.

6. Behere RV, John M, Sureshkumar R, Varambally S, Rao NP, Venkatasubramanian G, et al. Mood disorder in association with moyamoya disease. Psychiatry Clin Neurosci 2012;66:163-4.

7. Goyal JP, Rao SS, Trivedi S. Moya moya disease in a child: A case report. Case Rep Neurol Med 2011;2011:329738.

8. Sundaram S, Sylaja PN, Menon G, Sudhir J, Jayadevan ER, Sukumaran S, et al. Moyamoya disease: A comparison of long term outcome of conservative and surgical treatment in India. J Neurol Sci 2014;336:99-102.

This is an open access article distributed under the terms of the Creative Commons Attribution-NonCommercial-ShareAlike 3.0 License, which allows others to remix, tweak, and build upon the work non-commercially, as long as the author is credited and the new creations are licensed under the identical terms.

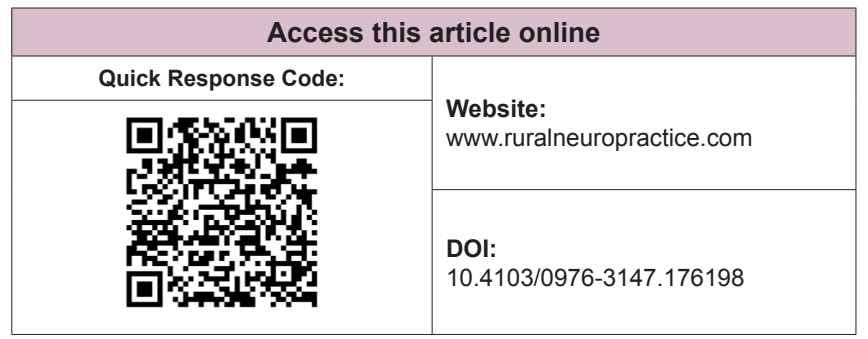

How to cite this article: Mohapatra S, Sahoo AJ. Neuropsychiatric manifestations in a child with moyamoya disease. J Neurosci Rural Pract 2016;7:331-2. 